\title{
Environmental and Genetic Variables Influencing Mitochondrial Health and Parkinson's Disease Penetrance
}

\author{
Alessandra Zanon, ${ }^{1}$ Peter P. Pramstaller, ${ }^{1,2,3}$ Andrew A. Hicks, ${ }^{1}$ and Irene Pichler $\mathbb{D}^{1}$ \\ ${ }^{1}$ Institute for Biomedicine, Eurac Research, Affiliated Institute of the University of Lübeck, Bolzano, Italy \\ ${ }^{2}$ Department of Neurology, General Central Hospital, Bolzano, Italy \\ ${ }^{3}$ Department of Neurology, University of Lübeck, Lübeck, Germany \\ Correspondence should be addressed to Irene Pichler; irene.pichler@eurac.edu
}

Received 10 October 2017; Accepted 7 December 2017; Published 7 March 2018

Academic Editor: Antonio Pisani

Copyright ( $\odot 2018$ Alessandra Zanon et al. This is an open access article distributed under the Creative Commons Attribution License, which permits unrestricted use, distribution, and reproduction in any medium, provided the original work is properly cited.

\begin{abstract}
There is strong evidence that impairment of mitochondrial function plays a key role in the pathogenesis of PD. The two key PD genes related to mitochondrial function are Parkin (PARK2) and PINK1 (PARK6), and also mutations in several other PD genes, including SNCA, LRRK2, DJ1, CHCHD2, and POLG, have been shown to induce mitochondrial stress. Many mutations are clearly pathogenic in some patients while carriers of other mutations either do not develop the disease or show a delayed onset, a phenomenon known as reduced penetrance. Indeed, for several mutations in autosomal dominant PD genes, penetrance is markedly reduced, whereas heterozygous carriers of recessive mutations may predispose to PD in a dominant manner, although with highly reduced penetrance, if additional disease modifiers are present. The identification and validation of such modifiers leading to reduced penetrance or increased susceptibility in the case of heterozygous carriers of recessive mutations are relevant for a better understanding of mechanisms contributing to disease onset. We discuss genetic and environmental factors as well as mitochondrial DNA alterations and protein-protein interactions, all involved in mitochondrial function, as potential causes to modify penetrance of mutations in dominant PD genes and to determine manifestation of heterozygous mutations in recessive PD genes.
\end{abstract}

\section{Introduction}

Parkinson's disease (PD) is the second most common neurodegenerative disease after Alzheimer's disease and is clinically defined as a motor syndrome consisting of levodopa responsive parkinsonism (bradykinesia, tremor, rigidity, and postural instability) and the absence of markers suggestive of other diseases [1]. It is characterized pathologically by loss of dopaminergic (DA) neurons in the substantia nigra pars compacta $(S N p c)$, loss of DA innervation in the striatum, and the presence of $\alpha$-synuclein positive aggregates (Lewy bodies). PD has an age-related prevalence, affecting around $1 \%$ of the population over the age of 65 , rising to up to $3 \%$ among individuals 80 years of age and older [2]. While most cases are thought to be sporadic, in about $10 \%$ of the patients, a genetic cause can be detected, ascribable to mutations in more than a dozen genes [3]. The rare monogenic forms clinically mimic the sporadic form and can thus serve as a disease model for this much more common form of PD. Mutations in several PD causing genes, encoding PINK1 (PTEN-induced serine/threonine kinase 1), Parkin, $\alpha$-synuclein, LRRK2 (leucine-rich repeat kinase 2), DJ1, CHCHD2 (coiled-coilhelix-coiled-coil-helix domain-containing 2), and POLG (mitochondrial DNA polymerase gamma), have been shown to induce mitochondrial dysfunction demonstrating that mitochondrial homeostasis and quality control have a central role in the disease process [4-14]. Several aspects of mitochondrial biology have been described as impaired in different PD models, leading to a defect in electron transport chain enzyme activities, ATP depletion, and generation of reactive oxygen species [15]. Systemic administration of the pesticides rotenone and paraquat as well as the neurotoxicant 
MPTP (1-methyl-4-phenyl-1,2,3,6-tetrahydropyridine), which are inhibitors of the mitochondrial respiratory chain $\mathrm{NADH}$ dehydrogenase (complex I), induces neuropathologic and behavioral changes in rodents similar to human PD [16, 17]. These data from basic science have been replicated in clinical studies showing a 20-30\% decrease of mitochondrial complex I activity in the $S N p c[18,19]$, in platelets $[20,21]$, and in lymphocytes of patients with sporadic PD [22], suggesting a systemic inhibition of complex I activity at least in a subset of the common form of PD. Aging is the biggest risk factor for $\mathrm{PD}$, and during aging mitochondrial function declines, with an accumulation of deletions in the mitochondrial DNA (mtDNA) [23, 24]. In line with these data, mtDNA depletion has been observed in nigral neurons from PD patients [25], but the mechanisms by which these mutations affect biochemical pathways leading to mitochondrial dysfunction are not answered yet. However, mtDNA levels or age-dependent increases in mtDNA mutations may be factors that influence penetrance of nuclear mutations known to act via mitochondrial mechanisms. While the phenomenon of reduced penetrance was initially described in the context of family studies, the advent of next-generation sequencing has revealed an unexpectedly large number of putatively pathogenic mutations in overtly healthy individuals, raising the need for a better understanding of factors influencing penetrance. Indeed, for several mutations in autosomal dominant PD genes penetrance is markedly reduced, whereas heterozygous carriers of mutations in recessive PD genes may also manifest disease or subclinical phenotypes of disease. In the following sections, we will review the evidence supporting the hypothesis that gene-environment, nuclear gene-mtDNA, and protein-protein interactions are able to modify the penetrance of genetic forms of PD.

\section{Environmental and Genetic Factors and Their Interplay as Modifiers of PD Penetrance}

For long time, environmental factors were thought to be the main cause of PD largely due to the epidemic of postencephalitic parkinsonism after the First World War [26]. Furthermore, this hypothesis was additionally supported by the identification of the mitochondrial neurotoxin MPTP in the early 1980s, which causes selective DA neuron degeneration by inhibiting mitochondrial respiratory electron transport chain complex I, leading to a parkinsonian syndrome in rodents, primates, and humans $[27,28]$. MPP+, the neurotoxic oxidation product of MPTP, concentrates in DA neurons of the $S N p c$ through the dopamine transporter, explaining the selective DA neuronal death $[29,30]$. Since then, several epidemiological and experimental studies have evaluated the role of a large number of environmental conditions and agents, including farming and rural life, industrial toxins, heavy metals, smoking, and drinking tea or coffee, in the pathogenesis of PD. Remarkably, a protective association was found for caffeine consumption [31] as well as for cigarette smoking, which could decrease the risk in a dosedependent manner [32]. On the contrary, epidemiological studies have described the exposure to pesticides, many known to inhibit electron transport chain activity, as an adverse risk factor for PD [33-36]. Importantly, the exposure to toxins like the pesticide rotenone and the herbicide paraquat reduced the activity of mitochondrial respiratory chain complex I and caused neurological defects similar to PD in humans and in animal models $[37,38]$. Chronic systemic administration of low doses of rotenone into a rat model induced nigrostriatal cell death and accumulation of proteinaceous inclusions similar to Lewy bodies [39]. However, data collected in another rotenone-based rat model did not support the generation of specific lesions of the $S N p c$ suggesting a generalized mitochondrial failure [40]. A mouse model with a deletion of the Ndufs 4 gene, encoding a subunit of complex I, and therefore having reduced complex I activity did not show significant DA neuron loss or motor impairment during lifespan, but showed a reduced amount of dopamine in the brain, increased $\alpha$-synuclein phosphorylation in DA neurons of the $S N p c$, and nonmotor symptoms including impaired cognitive function and increased anxiety-like behavior [41, 42]. These findings suggest that inhibition of complex I activity contributes to dopamine loss and $\alpha$-synuclein pathology and promotes nonmotor symptoms of $\mathrm{PD}$, but it is not sufficient to cause neurodegeneration during aging, suggesting the existence of additional susceptibility factors.

Of note, gene-environment interaction analyses have linked genetic variants in DAT/SLC6A3 likely affecting transport of chemicals into DA neurons to an increased risk for developing PD from exposures to the pesticides paraquat and maneb. Furthermore, variants in the metabolizing enzymes encoded by PON1 and NOS1 that may contribute to the nitrosative stress pathway were found to increase susceptibility to $\mathrm{PD}$ for organophosphate pesticide exposure. In addition, $A B C B 1$ gene variants that affect blood-brain barrier transport of chemicals increase organochlorine pesticides effect, and an association between pesticides inhibiting aldehyde dehydrogenase $(\mathrm{ALDH})$, involved in dopamine metabolism, with $A L D H 2$ gene variants was detected. These genes impact mitochondrial function via oxidative/nitrosative stress pathways and proteasome inhibition [43].

In addition to environmental and genetic risk factors, gene-environment interactions as well as aging as the main risk factors for developing $\mathrm{PD}$, intestinal microbiota have recently emerged as an additional factor able to promote $\alpha$-synuclein-induced motor deficits and microglia activation in the brain. Interestingly, transfer of microbiota from PD patients to $\alpha$-synuclein-overexpressing mice worsened motor impairments compared to microbiota from healthy individuals [44]. In line with these data, several studies have reported differences in the composition of gut microbiota between PD patients and controls [45-47]. While the major interest has been on the influence of such microbiota on the human immune system, there is some evidence that microbial metabolites (e.g., short chain fatty acids) influence mitochondrial function [48]. Relating the specific microbiomederived metabolites of PD patients to mitochondrial health is an exciting open research question, which will contribute to our knowledge of factors possibly influencing penetrance of PD. 


\section{Mitochondrial DNA Alterations and Reduced Penetrance of Nuclear PD Genes}

Human mtDNA is a small, circular 16,569 bp DNA encoding 13 subunits of mitochondrial respiratory chain proteins including seven complex I, one complex III, three complex IV, and two complex $\mathrm{V}$ subunits that are essential to the assembly and function of the mitochondrial respiratory chain $[49,50]$. Specific mutations in mtDNA have been found in patients with different forms of parkinsonism associated with mitochondrial disorders [51-53], and thus, mtDNA mutations are suspected to contribute to complex I deficiency in PD. Three different types of mtDNA alterations, mtDNA point mutations, mtDNA deletions, and mtDNA copy number have been investigated in postmortem brains or peripheral tissues like blood and skeletal muscle of PD patients. Specific mtDNA regions or the entire mtDNA genome were sequenced in several studies, and numerous point mutations were described [54-60]. However, no specific rare variant linked to PD was identified so far. There is conflicting reports in the literature describing the association between heteroplasmic variants and PD. Coxhead et al. found an increased mutational burden in both the $S N P C$ and frontal cortex of sporadic PD patients and a significant overrepresentation of PD cases harboring nonsynonymous heteroplasmic variants in MTCOX1, MTCOX2, and MTCYTB genes in the SNpc [61]. These findings could not be replicated in a recent study reporting a high frequency of heteroplasmy in the human brain, which did however not change with age or with PD disease state [60]. Furthermore, several groups have investigated the association of mtDNA haplogroups, aggregations of specific mtDNA variants, with PD. A reduced risk of PD was found for haplogroups J, $\mathrm{K}$, and $\mathrm{T}$, including a variant in the ND3 subunit of complex I, whereas the superhaplogroup HV showed an increased risk of PD [62, 63]. Moreover, not only heteroplasmic point mutations but also heteroplasmic deletions have been investigated, and a high proportion of multiple mtDNA deletions was found to accumulate in the SNPC and other brain regions with age, which was significantly more abundant in $\mathrm{PD}$ patients $[23,24]$. A recent study on human brain tissue convincingly showed a reduction of mtDNA copy number in single neurons of PD patients as compared to controls, which was more pronounced in neurons with severe complex I deficiency [64]. Interestingly, the MitoPark mouse, generated by direct deletion of mitochondrial transcription factor A (TFAM), which is needed for mtDNA replication, in DA neurons, is characterized by a marked deletion of mtDNA, impairment of oxidative phosphorylation, DA neuron degeneration, and motor deficits that mimic human parkinsonism [65]. These data provide evidence for a direct role of mtDNA alterations in mitochondrial function impairments.

As DA neurons are energetically highly demanding, mtDNA copy number or age-dependent increases in heteroplasmy might be factors that influence penetrance of nuclear mutations known also to act via mitochondrial mechanisms (e.g., Parkin and PINK1). Notably, in the mutator mouse model, characterized by the accumulation of multiple deletions in the mtDNA due to a proofreading-deficient form of POLG (the polymerase responsible for mtDNA replication), neuroprotective compensatory mechanisms at the mitochondrial level were observed [66]. After crossing this mouse with the Parkin knockout mouse, which also does not show neurodegeneration, mitochondrial dysfunction and PD pathology became apparent [67]. This study highlights a key role of high levels of mtDNA mutations in DA neurons and a protective role of Parkin, since its loss synergizes with mitochondrial dysfunction resulting in neurodegeneration.

Similar to POLG, mutations in other nuclear genes, like twinkle, a mtDNA helicase required for mtDNA replication and stability, lead to accumulation of mtDNA deletions and have been found to be associated with parkinsonism [68-70].

These studies provide examples for the association of mtDNA alterations, alone or in combination with nuclear gene mutations, with parkinsonism, and point to a role of mtDNA alterations in modifying penetrance of nuclear DNA mutations.

\section{Protein-Protein Interactions Regulate Mitochondrial Quality Control and Disease Penetrance}

By studying rare large families with a clear Mendelian inheritance, several causative genes have been identified, highlighting common intracellular functions involved in the pathogenesis of PD (mitochondria function and quality control (QC), lysosomal and endosomal pathways, synaptic transmission, and vesicle trafficking) [71]. In addition, genome-wide association studies have provided convincing evidence that polymorphic variants in these genes and 43 additional loci contribute to sporadic PD [72, 73]. Similar to other genetically complex diseases, these variants show only moderate effects on PD risk.

The most compelling evidence for the important role of mitochondrial function and QC in the $\mathrm{PD}$ pathogenesis has emerged by the elucidation of the function of the Parkin and PINK1 genes during the last decade. Studies in mammalian cells and the model organism Drosophila have demonstrated that Parkin, together with PINK1, regulates the degradation of dysfunctional mitochondria by the autophagy-lysosomal pathway, a process known as mitophagy [8,74-77]. Other $\mathrm{PD}$-linked proteins, like $\mathrm{FBXO} 7$ and VPS13C, might contribute to mitochondrial QC. An interaction of $\mathrm{FBXO} 7$ with PINK1 and Parkin contributes to mitochondrial maintenance through Parkin/PINK1-mediated mitophagy [78], and loss of function related to VPS13C exacerbates mitochondrial vulnerability to stress and increases PINK1/Parkin-dependent mitophagy [79].

Through direct or indirect interactions of Parkin/PINK1 with the fusion factors Mfn1, Mfn2, and OPA1 and the fission factor Drp1, these proteins regulate mitochondrial dynamics, which is linked to the maintenance of mitochondrial function [80-83]. Furthermore, Parkin and PINK1 have been implicated in another, autophagy-independent, mitochondrial QC mechanism, which is the transport of specific protein cargo as membrane-derived vesicles (MDVs) to the lysosomes. The formation of MDVs is stimulated as an early response to oxidative stress, and damaged mitochondrial regions are excised and transported to the endolysosomal compartments 


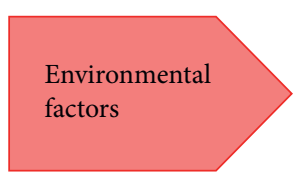

(i) Rotenone

(ii) Paraquat

(iii) MPTP
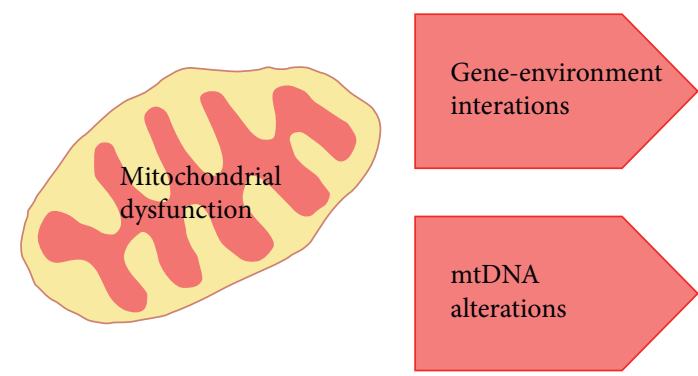

(i) DAT/SLC6A3: paraquat, maneb

(ii) PON1, NOS1: organophosphate pesticides

(iii) $A B C B 1$ : organochlorine pesticides

(iv) $A L D H 2$ : pesticides inhibiting aldehyde dehydrogenase (ALDH)

(v) Intestinal microbiota

(i) mtDNA point mutations

(ii) mtDNA deletions

(iii) mtDNA haplogroups

(iv) mtDNA copy number
(i) Parkin-PINK1
(ii) Parkin-PINK1-FBXO7
(iii) Parkin-PINK1-VPS13C
(iv) Parkin-PINK1-Mfn 1
(v) Parkin-PINK1-Mfn2
(vi) Parkin-PINK1-OPA1
(vii) Parkin-PINK1-Drp1
(viii) Parkin-PINK1-Miro
(ix) Parkin-PINK1-SLP-2

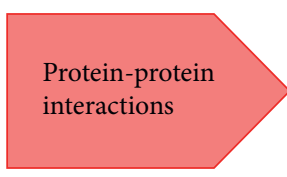

FIGURE 1: Modifying factors possibly underlying nonpenetrant or highly reduced penetrant forms of PD.

for degradation $[84,85]$. In addition to degradative pathways, Parkin and PINK1 proteins play a crucial role in the regulation of mitochondrial biogenesis through the degradation of the Parkin substrate PARIS, which acts as a transcriptional repressor of peroxisome proliferator-activated receptor gamma coactivator-1 alpha (PGC-1 $\alpha$ ), a transcriptional coactivator and master regulator of mitochondrial biogenesis [86, 87]. Moreover, an involvement of both Parkin and PINK1 in mitochondrial trafficking and QC has been suggested by reporting their association to Miro/Milton/dynein complex $[88,89]$. Miro is an outer mitochondrial membrane protein that anchors mitochondria to microtubule motors. As a consequence of Miro phosphorylation mediated by PINK1, Miro is ubiquitylated by Parkin and degraded by the proteasome. This leads to blockage of mitochondrial motility, which facilitates the isolation and sequestration of damaged mitochondria for degradation [88]. For example, an increase in anterograde transport was shown in axons of pink1 knockdown Drosophila [90], while mitochondrial trafficking was blocked by overexpression of both Parkin and Pink1 in Drosophila and in mammalian cells [88, 91]. More recently, it has been shown that also LRRK2 promotes Miro removal. The pathogenic G2019S mutation disrupts this function, delaying the motility blockage of damaged mitochondria and consequently slowing the initiation of mitophagy [92]. Remarkably, Miro degradation and reduced mitochondrial motility were also detected in sporadic PD patients, pointing to Miro and its interaction with PD-related proteins as a common molecular mechanism in different forms of PD. Recently, we have identified Stomatin-like protein 2 (SLP-2) as a novel Parkin interactor that upon overexpression rescued mitochondrial dysfunction of Parkin-deficient neuronal cells. Double knockdown flies showed a genetic interaction between Parkin and SLP-2, and SLP-2 transgenic flies attenuated loss of DA neurons, mitochondrial network structure, and flight and motor dysfunctions. This interaction might promote optimal activity of mitochondrial respiratory chain complex I and mitochondrial integrity in induced pluripotent stem cell-derived neurons and Drosophila [93]. SLP-2 was described as part of a new mitochondrial protein complex named SPY, which regulates proteolysis of PINK1 by the mitochondrial protease PARL linking SLP-2 to both Parkin and PINK1 [83].

Interactions between different $\mathrm{PD}$-causing proteins and/or their binding partners involved in mitochondrial QC might influence disease penetrance as basal and stress-evoked QC mechanisms might fail during ageing and/or the presence of mutations in genes encoding proteins involved in these pathways. The effects of the resulting mitotoxicity are deleterious and ultimately lead to degenerative processes specifically in vulnerable terminally differentiated neurons.

\section{Conclusions}

About $10 \%$ of all PD patients suffer from a monogenic form where autosomal dominant or recessive mutations in single genes are causative. Autosomal dominant PD is mostly represented by mutations in SNCA, coding for $\alpha$-synuclein, and LRRK2 and recessive PD by mutations in PINK1 and Parkin. Many of these mutations, particularly in the autosomal dominant forms, are only imperfectly penetrant or show a delay in the age at onset. Notably, heterozygous mutations in Parkin and PINK1 are relatively frequent (about $8 \%$ of all PD patients screened for Parkin mutations [94]) and may predispose to PD or subclinical phenotypes with highly reduced penetrance [95], although this remains 
controversial [96]. This reminds however mutations in the GBA gene (encoding the lysosomal enzyme glucocerebrosidase), which in a homozygous fashion, are responsible for the lysosomal storage disorder Gaucher's disease, while heterozygous mutations in this gene have been established as well-validated risk factors for PD $[97,98]$. The vast majority of $\mathrm{PD}$ patients are thought to be sporadic with a genetically complex form. In fact, more than 40 loci have been identified that are able to increase PD risk with however only modest to moderate effect size estimates (ORs ranging from 1.1 to 1 ) [72]. Given the high rate of reduced penetrance in PD, finding factors responsible for an incomplete or delayed disease onset and understanding the underlying mechanisms is important as they might be able to protect from developing the disease or delay the disease onset. We are highlighting environmental and genetic modifiers as well as mtDNA alterations and protein-protein interactions as possible factors underlying nonpenetrant or highly reduced penetrant forms of PD (Figure 1). These modifiers are all involved in the maintenance of mitochondrial function and may result in neuroprotection despite the presence of mutations causing PD in many instances. Validation of such modifiers would have high translational potential for the identification of early diagnosis and novel therapeutic options to halt the disease progression.

\section{Conflicts of Interest}

The authors declare that there are no conflicts of interests regarding the publication of this paper.

\section{Acknowledgments}

This work was partially supported by the Ministry of Health and Department of Educational Assistance, University and Research of the Autonomous Province of Bolzano, Italy and the DFG (FOR2488 Z2/Z1) to Andrew A. Hicks and Peter P. Pramstaller.

\section{References}

[1] D. Berg, R. B. Postuma, B. Bloem et al., "Time to redefine PD? Introductory statement of the MDS Task Force on the definition of Parkinson's disease," Movement Disorders, vol. 29, no. 4, pp. 454-462, 2014.

[2] C. M. Tanner and S. M. Goldman, "Epidemiology of Parkinson's disease," Neurologic Clinics, vol. 14, no. 2, pp. 317-335, 1996.

[3] A. Delamarre and W. G. Meissner, "Epidemiology, environmental risk factors and genetics of Parkinson's disease," $\mathrm{La}$ Presse Medicale, vol. 46, no. 2, pp. 175-181, 2017.

[4] N. Exner, B. Treske, D. Paquet et al., "Loss-of-function of human PINK1 results in mitochondrial pathology and can be rescued by parkin," Journal of Neuroscience, vol. 27, no. 45, pp. 12413-12418, 2007.

[5] A. Wood-Kaczmar, S. Gandhi, Z. Yao et al., "PINK1 is necessary for long term survival and mitochondrial function in human dopaminergic neurons," PLoS One, vol. 3, no. 6, article e2455, 2008.

[6] J. C. Greene, A. J. Whitworth, I. Kuo et al., "Mitochondrial pathology and apoptotic muscle degeneration in Drosophila parkin mutants," Proceedings of the National Academy of
Sciences of the United States of America, vol. 100, no. 7, pp. 4078-4083, 2003.

[7] J. J. Palacino, D. Sagi, M. S. Goldberg et al., "Mitochondrial dysfunction and oxidative damage in parkin-deficient mice," Journal of Biological Chemistry, vol. 279, no. 18, pp. 18614-18622, 2004.

[8] D. Narendra, A. Tanaka, D. F. Suen et al., "Parkin is recruited selectively to impaired mitochondria and promotes their autophagy," Journal of Cell Biology, vol. 183, no. 5, pp. 795-803, 2008.

[9] L. Devi, V. Raghavendran, B. M. Prabhu et al., "Mitochondrial import and accumulation of alpha-synuclein impair complex I in human dopaminergic neuronal cultures and Parkinson disease brain," Journal of Biological Chemistry, vol. 283, no. 14, pp. 9089-9100, 2008.

[10] X. Wang, M. H. Yan, H. Fujioka et al., "LRRK2 regulates mitochondrial dynamics and function through direct interaction with DLP1," Human Molecular Genetics, vol. 21, no. 9, pp. 1931-1944, 2012.

[11] M. Funayama, K. Ohe, T. Amo et al., "CHCHD2 mutations in autosomal dominant late-onset Parkinson's disease: a genomewide linkage and sequencing study," The Lancet Neurology, vol. 14, no. 3, pp. 274-282, 2015.

[12] C. H. Shi, C. Y. Mao, S. Y. Zhang et al., "CHCHD2 gene mutations in familial and sporadic Parkinson's disease," Neurobiology of Aging, vol. 38, pp. 217 e219-217 e213, 2016.

[13] A. M. Remes, R. Hinttala, M. Karppa et al., "Parkinsonism associated with the homozygous W748S mutation in the POLG1 gene," Parkinsonism \& Related Disorders, vol. 14, no. 8, pp. 652-654, 2008.

[14] M. Delgado-Alvarado, P. de la Riva, H. Jimenez-Urbieta et al., "Parkinsonism, cognitive deficit and behavioural disturbance caused by a novel mutation in the polymerase gamma gene," Journal of the Neurological Sciences, vol. 350, no. 1-2, pp. 93-97, 2015.

[15] N. Exner, A. K. Lutz, C. Haass et al., "Mitochondrial dysfunction in Parkinson's disease: molecular mechanisms and pathophysiological consequences," EMBO Journal, vol. 31, no. 14, pp. 3038-3062, 2012.

[16] E. Bezard and S. Przedborski, "A tale on animal models of Parkinson's disease," Movement Disorders, vol. 26, no. 6, pp. 993-1002, 2011.

[17] J. R. Cannon and J. T. Greenamyre, "The role of environmental exposures in neurodegeneration and neurodegenerative diseases," Toxicological Sciences, vol. 124, no. 2, pp. 225-250, 2011.

[18] A. H. Schapira, J. M. Cooper, D. Dexter et al., "Mitochondrial complex I deficiency in Parkinson's disease," Journal of Neurochemistry, vol. 54, no. 3, pp. 823-827, 1990.

[19] Y. Mizuno, S. Ohta, M. Tanaka et al., "Deficiencies in complex I subunits of the respiratory chain in Parkinson's disease," Biochemical and Biophysical Research Communications, vol. 163, no. 3, pp. 1450-1455, 1989.

[20] W. D. Parker, S. J. Boyson, and J. K. Parks, "Abnormalities of the electron transport chain in idiopathic Parkinson's disease," Annals of Neurology, vol. 26, no. 6, pp. 719-723, 1989.

[21] R. H. Haas, F. Nasirian, K. Nakano et al., "Low platelet mitochondrial complex I and complex II/III activity in early untreated Parkinson's disease," Annals of Neurology, vol. 37, no. 6, pp. 714-722, 1995.

[22] N. Barroso, Y. Campos, R. Huertas et al., "Respiratory chain enzyme activities in lymphocytes from untreated patients with Parkinson disease," Clinical Chemistry, vol. 39, no. 4, pp. 667-669, 1993. 
[23] A. Bender, K. J. Krishnan, C. M. Morris et al., "High levels of mitochondrial DNA deletions in substantia nigra neurons in aging and Parkinson disease," Nature Genetics, vol. 38, no. 5, pp. 515-517, 2006.

[24] Y. Kraytsberg, E. Kudryavtseva, A. C. McKee, C. Geula, N. W. Kowall, and K. Khrapko, "Mitochondrial DNA deletions are abundant and cause functional impairment in aged human substantia nigra neurons," Nature Genetics, vol. 38, no. 5, pp. 518-520, 2006.

[25] C. Dolle, I. Flones, G. S. Nido et al., "Defective mitochondrial DNA homeostasis in the substantia nigra in Parkinson disease," Nature Communications, vol. 7, p. 13548, 2016.

[26] J. Casals, T. S. Elizan, and M. D. Yahr, "Postencephalitic parkinsonism-a review," Journal of Neural Transmission, vol. 105, no. 6-7, pp. 645-676, 1998.

[27] J. W. Langston, P. Ballard, J. W. Tetrud, and I. Irwin, "Chronic Parkinsonism in humans due to a product of meperidineanalog synthesis," Science, vol. 219, no. 4587, pp. 979-980, 1983.

[28] T. P. Singer and R. R. Ramsay, "Mechanism of the neurotoxicity of MPTP. An update," FEBS Letters, vol. 274, no. 1-2, pp. 1-8, 1990.

[29] J. A. Javitch, R. J. D'Amato, S. M. Strittmatter, and S. H. Snyder, "Parkinsonism-inducing neurotoxin, N-methyl4-phenyl-1,2,3,6 -tetrahydropyridine: uptake of the metabolite $\mathrm{N}$-methyl-4-phenylpyridine by dopamine neurons explains selective toxicity," Proceedings of the National Academy of Sciences of the United States of America, vol. 82, no. 7, pp. 2173-2177, 1985.

[30] R. R. Ramsay and T. P. Singer, "Energy-dependent uptake of $\mathrm{N}$-methyl-4-phenylpyridinium, the neurotoxic metabolite of 1-methyl-4-phenyl-1,2,3,6-tetrahydropyridine, by mitochondria," Journal of Biological Chemistry, vol. 261, no. 17, pp. 7585-7587, 1986.

[31] M. A. Hernan, B. Takkouche, F. Caamano-Isorna, and J. J. Gestal-Otero, "A meta-analysis of coffee drinking, cigarette smoking, and the risk of Parkinson's disease," Annals of Neurology, vol. 52, no. 3, pp. 276-284, 2002.

[32] B. Ritz, A. Ascherio, H. Checkoway et al., "Pooled analysis of tobacco use and risk of Parkinson disease," Archives of Neurology, vol. 64, no. 7, pp. 990-997, 2007.

[33] J. R. Richardson, S. L. Shalat, B. Buckley et al., "Elevated serum pesticide levels and risk of Parkinson disease," Archives of Neurology, vol. 66, no. 7, pp. 870-875, 2009.

[34] R. A. Schuh, T. Kristian, R. K. Gupta, J. A. Flaws, and G. Fiskum, "Methoxychlor inhibits brain mitochondrial respiration and increases hydrogen peroxide production and CREB phosphorylation," Toxicological Sciences, vol. 88, no. 2, pp. 495-504, 2005.

[35] R. A. Schuh, J. R. Richardson, R. K. Gupta, J. A. Flaws, and G. Fiskum, "Effects of the organochlorine pesticide methoxychlor on dopamine metabolites and transporters in the mouse brain," Neurotoxicology, vol. 30, no. 2, pp. 274-280, 2009.

[36] T. B. Sherer, R. Betarbet, and J. T. Greenamyre, "Environment, mitochondria, and Parkinson's disease," Neuroscientist, vol. 8, no. 3, pp. 192-197, 2002.

[37] J. Bove and C. Perier, "Neurotoxin-based models of Parkinson's disease," Neuroscience, vol. 211, pp. 51-76, 2012.

[38] A. H. Schapira, "Mitochondria in the aetiology and pathogenesis of Parkinson's disease," The Lancet Neurology, vol. 7, no. 1, pp. 97-109, 2008.

[39] R. Betarbet, T. B. Sherer, G. MacKenzie, M. Garcia-Osuna, A. V. Panov, and J. T. Greenamyre, "Chronic systemic pesticide exposure reproduces features of Parkinson's disease," Nature Neuroscience, vol. 3, no. 12, pp. 1301-1306, 2000.

[40] G. U. Hoglinger, J. Feger, A. Prigent et al., "Chronic systemic complex I inhibition induces a hypokinetic multisystem degeneration in rats," Journal of Neurochemistry, vol. 84, no. 3, pp. 491-502, 2003.

[41] H. W. Kim, W. S. Choi, N. Sorscher et al., "Genetic reduction of mitochondrial complex I function does not lead to loss of dopamine neurons in vivo," Neurobiology of Aging, vol. 36, no. 9, pp. 2617-2627, 2015.

[42] W. S. Choi, H. W. Kim, F. Tronche, R. D. Palmiter, D. R. Storm, and Z. Xia, "Conditional deletion of Ndufs4 in dopaminergic neurons promotes Parkinson's disease-like non-motor symptoms without loss of dopamine neurons," Scientific Reports, vol. 7, p. 44989, 2017.

[43] B. R. Ritz, K. C. Paul, and J. M. Bronstein, "Of pesticides and men: a California story of genes and environment in Parkinson's disease," Current Environmental Health Reports, vol. 3, no. 1, pp. 40-52, 2016.

[44] T. R. Sampson, J. W. Debelius, T. Thron et al., "Gut microbiota regulate motor deficits and neuroinflammation in a model of Parkinson's disease," Cell, vol. 167, no. 6, pp. 1469-1480 e12, 2016.

[45] F. Scheperjans, V. Aho, P. A. Pereira et al., "Gut microbiota are related to Parkinson's disease and clinical phenotype," Movement Disorders, vol. 30, no. 3, pp. 350-358, 2015.

[46] M. M. Unger, J. Spiegel, K. U. Dillmann et al., "Short chain fatty acids and gut microbiota differ between patients with Parkinson's disease and age-matched controls," Parkinsonism \& Related Disorders, vol. 32, pp. 66-72, 2016.

[47] E. M. Hill-Burns, J. W. Debelius, J. T. Morton et al., "Parkinson's disease and Parkinson's disease medications have distinct signatures of the gut microbiome," Movement Disorders, vol. 32, no. 5, pp. 739-749, 2017.

[48] P. Bajpai, A. Darra, and A. Agrawal, "Microbe-mitochondrion crosstalk and health: an emerging paradigm," Mitochondrion, 2017, in press.

[49] S. Anderson, A. T. Bankier, B. G. Barrell et al., "Sequence and organization of the human mitochondrial genome," Nature, vol. 290, no. 5806, pp. 457-465, 1981.

[50] S. DiMauro and E. A. Schon, "Mitochondrial respiratorychain diseases," New England Journal of Medicine, vol. 348, no. 26, pp. 2656-2668, 2003.

[51] D. K. Simon, S. M. Pulst, J. P. Sutton, S. E. Browne, M. F. Beal, and D. R. Johns, "Familial multisystem degeneration with parkinsonism associated with the 11778 mitochondrial DNA mutation," Neurology, vol. 53, no. 8, pp. 1787-1793, 1999.

[52] D. Thyagarajan, S. Bressman, C. Bruno et al., "A novel mitochondrial 12SrRNA point mutation in parkinsonism, deafness, and neuropathy," Annals of Neurology, vol. 48, no. 5, pp. 730-736, 2000.

[53] M. Rana, I. de Coo, F. Diaz, H. Smeets, and C. T. Moraes, “An out-of-frame cytochrome b gene deletion from a patient with parkinsonism is associated with impaired complex III assembly and an increase in free radical production," Annals of Neurology, vol. 48, no. 5, pp. 774-781, 2000.

[54] R. M. Kapsa, M. J. Jean-Francois, P. Lertrit et al., "Mitochondrial DNA polymorphism in substantia nigra," Journal of the Neurological Sciences, vol. 144, no. 1-2, pp. 204-211, 1996.

[55] S. Kosel, E. M. Grasbon-Frodl, U. Mautsch et al., "Novel mutations of mitochondrial complex I in pathologically proven Parkinson disease," Neurogenetics, vol. 1, no. 3, pp. 197-204, 1998.

[56] D. K. Simon, R. Mayeux, K. Marder, N. W. Kowall, M. F. Beal, and D. R. Johns, "Mitochondrial DNA mutations in complex I 
and tRNA genes in Parkinson's disease," Neurology, vol. 54, no. 3, pp. 703-709, 2000.

[57] S. Ikebe, M. Tanaka, and T. Ozawa, "Point mutations of mitochondrial genome in Parkinson's disease," Molecular Brain Research, vol. 28, no. 2, pp. 281-295, 1995.

[58] M. D. Brown, J. M. Shoffner, Y. L. Kim et al., "Mitochondrial DNA sequence analysis of four Alzheimer's and Parkinson's disease patients," American Journal of Medical Genetics, vol. 61, no. 3, pp. 283-289, 1996.

[59] C. Vives-Bauza, A. L. Andreu, G. Manfredi et al., "Sequence analysis of the entire mitochondrial genome in Parkinson's disease," Biochemical and Biophysical Research Communications, vol. 290, no. 5, pp. 1593-1601, 2002.

[60] W. Wei, M. J. Keogh, I. Wilson et al., "Mitochondrial DNA point mutations and relative copy number in 1363 disease and control human brains," Acta Neuropathologica Communications, vol. 5, no. 1, p. 13, 2017.

[61] J. Coxhead, M. Kurzawa-Akanbi, R. Hussain, A. Pyle, P. Chinnery, and G. Hudson, "Somatic mtDNA variation is an important component of Parkinson's disease," Neurobiology of Aging, vol. 38, pp. 217 e1-217 e6, 2016.

[62] J. M. van der Walt, K. K. Nicodemus, E. R. Martin et al., "Mitochondrial polymorphisms significantly reduce the risk of Parkinson disease," American Journal of Human Genetics, vol. 72, no. 4, pp. 804-811, 2003.

[63] G. Hudson, M. Nalls, J. R. Evans et al., "Two-stage association study and meta-analysis of mitochondrial DNA variants in Parkinson disease," Neurology, vol. 80, no. 22, pp. 2042-2048, 2013.

[64] A. Grunewald, K. A. Rygiel, P. D. Hepplewhite, C. M. Morris, M. Picard, and D. M. Turnbull, "Mitochondrial DNA depletion in respiratory chain-deficient Parkinson disease neurons," Annals of Neurology, vol. 79, no. 3, pp. 366-378, 2016.

[65] M. I. Ekstrand, M. Terzioglu, D. Galter et al., "Progressive parkinsonism in mice with respiratory-chain-deficient dopamine neurons," Proceedings of the National Academy of Sciences of the United States of America, vol. 104, no. 4, pp. 1325-1330, 2007.

[66] C. Perier, A. Bender, E. Garcia-Arumi et al., "Accumulation of mitochondrial DNA deletions within dopaminergic neurons triggers neuroprotective mechanisms," Brain, vol. 136, no. 8, pp. 2369-2378, 2013.

[67] A. M. Pickrell, C. H. Huang, S. R. Kennedy et al., "Endogenous parkin preserves dopaminergic substantia nigral neurons following mitochondrial DNA mutagenic stress," Neuron, vol. 87, no. 2, pp. 371-381, 2015.

[68] G. Davidzon, P. Greene, M. Mancuso et al., "Early-onset familial parkinsonism due to POLG mutations," Annals of Neurology, vol. 59, no. 5, pp. 859-862, 2006.

[69] R. H. Baloh, E. Salavaggione, J. Milbrandt, and A. Pestronk, "Familial parkinsonism and ophthalmoplegia from a mutation in the mitochondrial DNA helicase twinkle," Archives of Neurology, vol. 64, no. 7, pp. 998-1000, 2007.

[70] V. Carelli and D. C. Chan, "Mitochondrial DNA: impacting central and peripheral nervous systems," Neuron, vol. 84, no. 6, pp. 1126-1142, 2014.

[71] O. Corti, S. Lesage, and A. Brice, "What genetics tells us about the causes and mechanisms of Parkinson's disease," Physiological Reviews, vol. 91, no. 4, pp. 1161-1218, 2011.

[72] C. M. Lill, "Genetics of Parkinson's disease," Molecular and Cellular Probes, vol. 30, no. 6, pp. 386-396, 2016.

[73] D. Chang, M. A. Nalls, I. B. Hallgrimsdottir et al., "A metaanalysis of genome-wide association studies identifies 17 new
Parkinson's disease risk loci," Nature Genetics, vol. 49, no. 10, pp. 1511-1516, 2017.

[74] I. E. Clark, M. W. Dodson, C. Jiang et al., "Drosophila pink1 is required for mitochondrial function and interacts genetically with parkin," Nature, vol. 441, no. 7097, pp. 1162-1166, 2006.

[75] J. Park, S. B. Lee, S. Lee et al., "Mitochondrial dysfunction in Drosophila PINK1 mutants is complemented by parkin," Nature, vol. 441, no. 7097, pp. 1157-1161, 2006.

[76] S. Geisler, K. M. Holmstrom, D. Skujat et al., "PINK1/Parkinmediated mitophagy is dependent on VDAC1 and p62/SQSTM1," Nature Cell Biology, vol. 12, no. 2, pp. 119-131, 2010.

[77] C. Vives-Bauza, C. Zhou, Y. Huang et al., "PINK1-dependent recruitment of Parkin to mitochondria in mitophagy," Proceedings of the National Academy of Sciences of the United States of America, vol. 107, no. 1, pp. 378-383, 2010.

[78] V. S. Burchell, D. E. Nelson, A. Sanchez-Martinez et al., "The Parkinson's disease-linked proteins Fbxo7 and Parkin interact to mediate mitophagy," Nature Neuroscience, vol. 16, no. 9, pp. 1257-1265, 2013.

[79] S. Lesage, V. Drouet, E. Majounie et al., "Loss of VPS13C function in autosomal-recessive parkinsonism causes mitochondrial dysfunction and increases PINK1/Parkin-dependent mitophagy," American Journal of Human Genetics, vol. 98, no. 3, pp. 500-513, 2016.

[80] M. E. Gegg, J. M. Cooper, K. Y. Chau, M. Rojo, A. H. V. Schapira, and J.-W. Taanman, "Mitofusin 1 and mitofusin 2 are ubiquitinated in a PINK1/Parkin-dependent manner upon induction of mitophagy," Human Molecular Genetics, vol. 19, no. 24, pp. 4861-4870, 2010.

[81] A. Tanaka, M. M. Cleland, S. Xu et al., "Proteasome and p97 mediate mitophagy and degradation of mitofusins induced by parkin," Journal of Cell Biology, vol. 191, no. 7, pp. 1367-1380, 2010.

[82] E. Ziviani, R. N. Tao, and A. J. Whitworth, "Drosophila parkin requires PINK1 for mitochondrial translocation and ubiquitinates mitofusin," Proceedings of the National Academy of Sciences of the United States of America, vol. 107, no. 11, pp. 5018-5023, 2010.

[83] T. Wai, S. Saita, H. Nolte et al., "The membrane scaffold SLP2 anchors a proteolytic hub in mitochondria containing PARL and the i-AAA protease YME1L," EMBO Reports, vol. 17, no. 12, pp. 1844-1856, 2016.

[84] V. Soubannier, G. L. McLelland, R. Zunino et al., "A vesicular transport pathway shuttles cargo from mitochondria to lysosomes," Current Biology, vol. 22, no. 2, pp. 135-141, 2012.

[85] G. L. McLelland, V. Soubannier, C. X. Chen et al., "Parkin and PINK1 function in a vesicular trafficking pathway regulating mitochondrial quality control," EMBO Journal, vol. 33, no. 4, pp. 282-295, 2014.

[86] J. H. Shin, H. S. Ko, H. Kang et al., "PARIS (ZNF746) repression of PGC-1a contributes to neurodegeneration in Parkinson's disease," Cell, vol. 144, no. 5, pp. 689-702, 2011.

[87] Y. Lee, D. A. Stevens, S. U. Kang et al., "PINK1 primes parkinmediated ubiquitination of PARIS in dopaminergic neuronal survival," Cell Reports, vol. 18, no. 4, pp. 918-932, 2017.

[88] X. Wang, D. Winter, G. Ashrafi et al., "PINK1 and Parkin target Miro for phosphorylation and degradation to arrest mitochondrial motility," Cell, vol. 147, no. 4, pp. 893-906, 2011.

[89] A. Weihofen, K. J. Thomas, B. L. Ostaszewski, M. R. Cookson, and D. J. Selkoe, "Pink1 forms a multiprotein complex with Miro and Milton, linking Pink1 function to mitochondrial trafficking," Biochemistry, vol. 48, no. 9, pp. 2045-2052, 2009. 
[90] S. Liu, T. Sawada, S. Lee et al., "Parkinson's disease-associated kinase PINK1 regulates Miro protein level and axonal transport of mitochondria," PLoS Genetics, vol. 8, no. 3, p. e1002537, 2012.

[91] N. C. Chan, A. M. Salazar, A. H. Pham et al., "Broad activation of the ubiquitin-proteasome system by Parkin is critical for mitophagy," Human Molecular Genetics, vol. 20, no. 9, pp. 1726-1737, 2011.

[92] C. H. Hsieh, A. Shaltouki, A. E. Gonzalez et al., "Functional impairment in Miro degradation and mitophagy is a shared feature in familial and sporadic Parkinson's disease," Cell Stem Cell, vol. 19, no. 6, pp. 709-724, 2016.

[93] A. Zanon, S. Kalvakuri, A. Rakovic et al., "SLP-2 interacts with Parkin in mitochondria and prevents mitochondrial dysfunction in Parkin-deficient human iPSC-derived neurons and Drosophila," Human Molecular Genetics, vol. 26, no. 13, pp. 2412-2425, 2017.

[94] C. Klein, K. Lohmann-Hedrich, E. Rogaeva, M. G. Schlossmacher, and A. E. Lang, "Deciphering the role of heterozygous mutations in genes associated with parkinsonism," The Lancet Neurology, vol. 6, no. 7, pp. 652-662, 2007.

[95] E. M. Valente, S. Salvi, T. Ialongo et al., "PINK1 mutations are associated with sporadic early-onset parkinsonism," Annals of Neurology, vol. 56, no. 3, pp. 336-341, 2004.

[96] D. M. Kay, C. F. Stevens, T. H. Hamza et al., "A comprehensive analysis of deletions, multiplications, and copy number variations in PARK2," Neurology, vol. 75, no. 13, pp. 1189-1194, 2010.

[97] E. Sidransky, M. A. Nalls, J. O. Aasly et al., "Multicenter analysis of glucocerebrosidase mutations in Parkinson's disease," New England Journal of Medicine, vol. 361, no. 17, pp. 1651-1661, 2009.

[98] F. Zhao, L. Bi, W. Wang et al., "Mutations of glucocerebrosidase gene and susceptibility to Parkinson's disease: an updated meta-analysis in a European population," Neuroscience, vol. 320, pp. 239-246, 2016. 


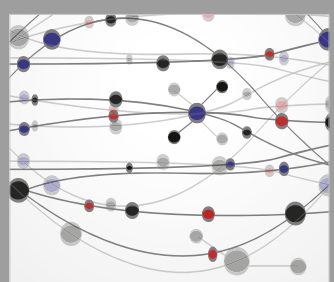

The Scientific World Journal
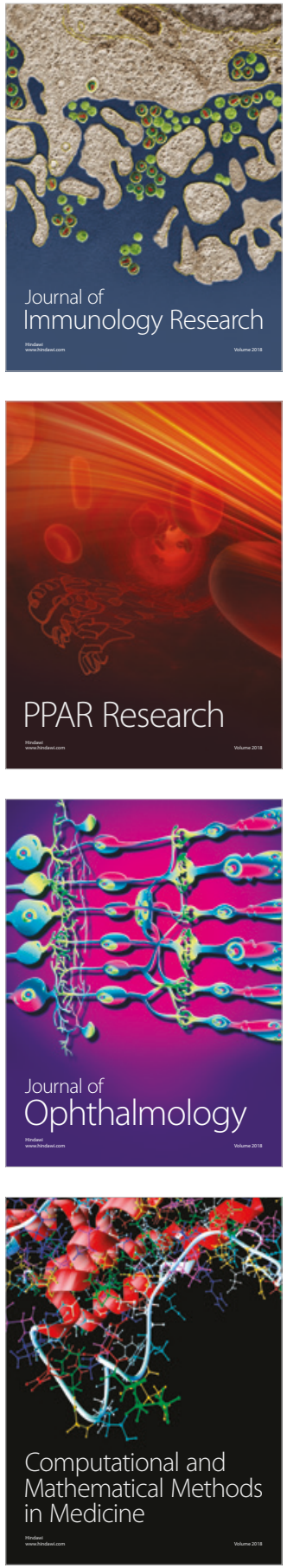

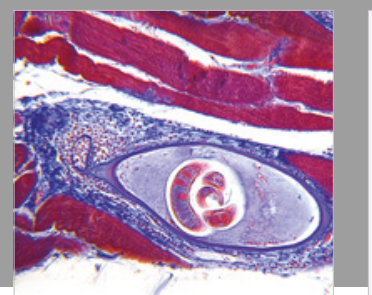

Gastroenterology Research and Practice

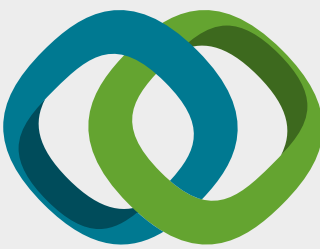

\section{Hindawi}

Submit your manuscripts at

www.hindawi.com
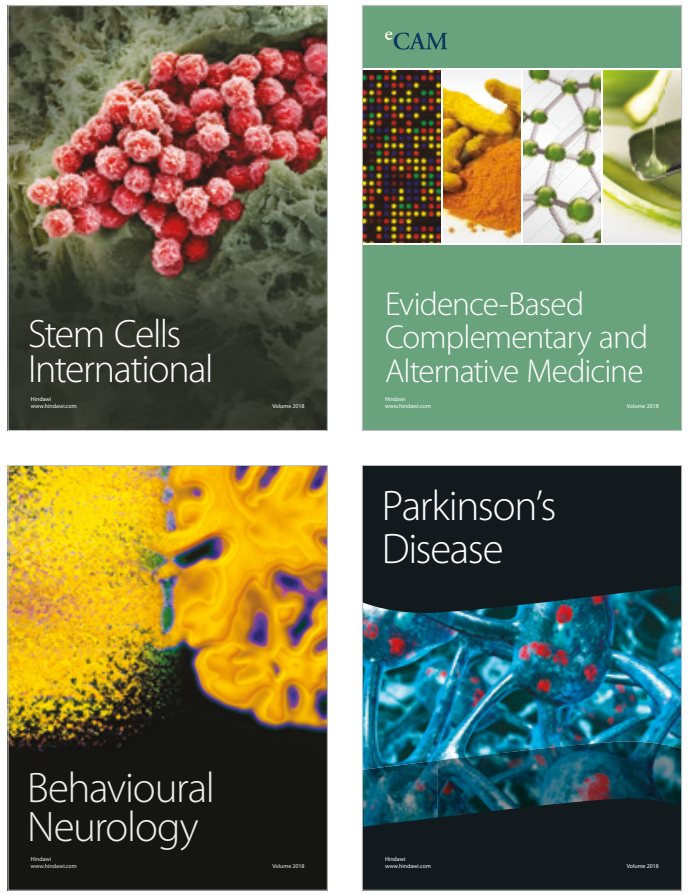

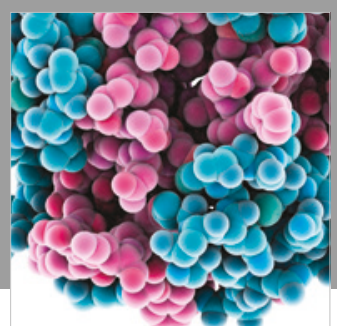

ournal of

Diabetes Research

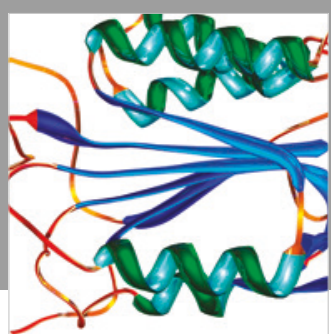

Disease Markers
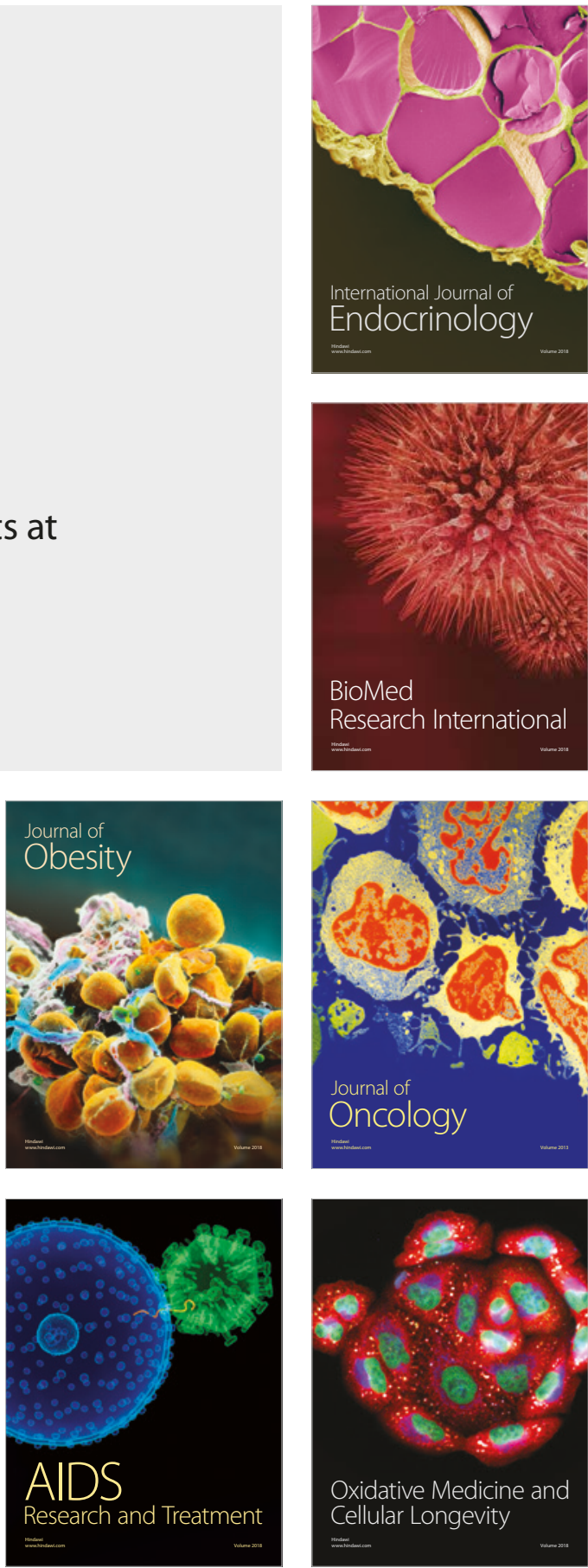